\title{
Entre protection et mobilisation : contributions canadiennes et québécoises à l'histoire transnationale des droits des enfants handicapés (1920-1980)
}

\author{
Susanne Commend \\ Postdoctoral fellow at the Institute of Feminist and Gender Studies \\ University of Ottawa
}

\begin{abstract}
Résumé
Cet article repère les principaux jalons de la mobilisation à l'égard des enfants handicapés au Québec et dans le monde. Il s'intéresse en particulier à l'émergence d'un mouvement de défense des droits des enfants infirmes sur la scène internationale pendant l'entre-deux-guerres, auquel le Canada, incluant le Québec, ont participé. En s'appuyant sur un corpus de sources variées, l'étude démontre qu'une conception sociale de l'enfance «infirme» a principalement été véhiculée par les acteurs de ce mouvement, sans être pour autant exempte de l'influence médicale. En insistant sur les causes socioéconomiques responsables de l'infirmité, les philanthropes discréditent un autre discours qui fait porter le poids des déficiences sur des facteurs individuels. Notre lecture de ce mouvement d'avant-garde nuance l'interprétation selon laquelle une emprise médicale hégémonique aurait pesé sur les jeunes handicapés jusqu'à l'intensification de l'activisme dans les années 1960. L'article distingue trois périodes de mobilisation, sans exclure des recoupements entre ces bornes chronologiques : un premier mouvement de défense des droits des jeunes infirmes voit le jour pendant l'entre-deux-guerres et s'estompe à la veille de la Deuxième Guerre mondiale. Cette promotion des droits connaît un second souffle dans l'après-guerre jusqu'aux années 1960, et enfin une troisième vague de mobilisation s'intensifie des années 1960 au milieu des années 1980.
\end{abstract}

Mots-clefs : Enfants handicapés, Québec, droits des enfants, mobilisation, philanthropie, mouvements sociaux, histoire transnationale.

\begin{abstract}
:
This article identifies the main milestones in the mobilization of children with disabilities in Quebec and around the world. The paper is particularly interested in the emergence of a movement to defend the rights of disabled children on the international scene during the inter-war period, in which Canada, including Quebec, participated. Based on a body of varied sources, the study shows that a social conception of «crippled» childhood was mainly conveyed by the actors of this movement, without being free from medical influence. By emphasizing the socio-economic causes of disability, philanthropists discredit another discourse that places the burden of disability on individual factors. Our reading of this pioneering movement nuances the interpretation that a hegemonic medical hold would have weighed on young people with disabilities until the intensification of activism in the 1960s. The article distinguishes three periods of mobilization, without
\end{abstract}


excluding overlaps between these chronological boundaries: a first movement for the defense of the rights of disabled children starts during the inter-war period and fades on the eve of the Second World War World. This promotion of rights has a second wind in the post-war period until the 1960s, and finally a third wave of mobilization intensifies from the 1960s to the mid-1980s.

Key words: Disabled children, Québec, children's rights, mobilization, philanthropy, social movements, transnational history.

\section{Introduction}

Cet article vise à repérer les principaux jalons de la défense des droits des enfants handicapés dans le monde et décrit l'émergence d'une prise de conscience de la situation des enfants «infirmes» sur la scène internationale pendant l'entre-deux-guerres. Il tente de démontrer les liens entre les nouvelles sensibilités à l'égard de l'enfance et l'éveil des consciences quant aux conditions de vie des jeunes handicapés, en se penchant aussi sur la participation du Québec à ce mouvement transnational. Campée dans le courant des études critiques sur le handicap, l'approche considère le handicap comme une construction culturelle et sociale qui fluctue à travers le temps. La démarche vise aussi à «désinsulariser» le handicap (Gardou, 2005), en intégrant ce marqueur identitaire au cœur de l'analyse, de la même manière que le genre, l'ethnie, la classe sociale ou l'âge s'amalgament à ce récit plus vaste ${ }^{1}$.

Située à l'intersection du domaine de la protection de l'enfance et des politiques publiques du handicap, l'étude s'inscrit aussi dans la foulée de l'historiographie consacrée à l'étude des réseaux transnationaux. Comme l'a minutieusement démontré Gildas Brégain en s'inspirant de la thèse développée par Pierre-Yves Saunier, «les normes et standards internationaux à suivre en matière de politiques publiques du handicap sont définis - parfois de manière conflictuelle- à la fois par les organismes intergouvernementaux et par les multiples ONG concernées. Leur action contribue à l'institutionnalisation de savoirs, de techniques, et d'institutions considérés désormais comme universelles» $\rangle^{2}$. Notre recherche décrit quelques moments forts de la circulation des idées et de ces échanges transnationaux animés par divers acteurs sur la question des droits des jeunes handicapés, sans toutefois en reconstituer le récit détaillé, notamment en raison de la documentation lacunaire retrouvée dans les fonds d'archives canadiens et québécois et parce que nous n'avons pas pu élargir l'investigation aux archives de la 
Société des nations. Le croisement de sources fragmentaires, notamment les archives de la Fondation Papillon ${ }^{3}$, laissées dans l'ombre jusqu'ici, avec certains documents retrouvés dans le fonds de la Commission scolaire de Montréal nous ont néanmoins révélé des informations inattendues sur la participation des philanthropes québécois à la mobilisation transnationale autour de l'«enfance infirme» pendant l'entre-deux-guerres.

L'argumentation soutient que pendant l'entre-deux-guerres, une conception sociale de l'enfance infirme a principalement été véhiculée par les tenants du mouvement de défense des droits de ces enfants, sans être pour autant exempte de l'influence médicale. Cette perception tranche alors sur d'autres théories de l'époque, comme celle de la dégénérescence qui fait porter le poids des déficiences sur l'hérédité ou des causes strictement individuelles, plutôt que socio-économiques. L'analyse relève les tensions entre la sphère médicale et la sphère sociale, ainsi que les contradictions entre les discours exprimés par les divers acteurs. Ainsi, certaines revendications ayant de prime abord une portée sociale sont parfois aussi chargées d'un relent d'eugénisme. Dans la foulée de travaux récents, cette perspective historique nuance néanmoins la thèse de l'emprise médicale hégémonique qui aurait pesé sur les personnes handicapées jusqu'à l'affirmation du modèle social ${ }^{4}$ dans les années 1970.

L'article distingue trois périodes de mobilisation, sans exclure des recoupements entre ces bornes chronologiques : un premier mouvement de défense des droits des jeunes infirmes voit le jour pendant l'entre-deux-guerres et s'estompe à la veille de la Deuxième Guerre mondiale. Cette promotion des droits connaît un second souffle dans l'aprèsguerre jusqu'aux années 1960, et enfin une troisième vague de mobilisation s'intensifie des années 1960 au début des années 1980, aboutissant à la proclamation de l'Année internationale des personnes handicapées en 1981. À chacune de ces périodes correspond une terminologie distincte pour identifier ces enfants, délibérément reprise dans cette recherche puisqu'elle renvoie à un schème de pensées, à une manière collective d'entrevoir et de conceptualiser l'enfance «irrégulière». Ainsi aux qualificatifs d'«infirmes» ou d'«estropiés» succèdent celui d'«exceptionnels» puis d'«inadaptés», pour qu'enfin se substitue à ces diverses appellations « le vocabulaire du handicap» (Stiker, 2013). 


\section{Nommer et conceptualiser les déficiences : critères et catégories mouvantes}

Pendant l'entre-deux-guerres, le terme «infirme», qui évoque la faiblesse, figure dans les archives des associations philanthropiques du Québec, alors qu'en Europe, le terme «estropié», signifiant la privation de l'usage d'un membre par blessure ou par maladie, semble davantage privilégié ${ }^{5}$. Cette image du corps blessé est sans doute liée aux ravages de la Grande Guerre sur le vieux continent. Dans les années 1950, le terme «enfance exceptionnelle», calque du terme anglophone exceptional children en usage aux États-Unis, est utilisé par les spécialistes québécois oeuvrant auprès de cette clientèle. Au milieu des années 1960 jusqu'à la décennie 1970 s'impose par la suite le mot «inadapté» sous l'influence des collègues franco-européens (Ferreti, Bienvenue, 2010). Tout comme l'anormalité, la notion d'inadaptation réfère à un écart, et même dans ce cas à une infériorité face à la norme, contribuant à catégoriser les enfants selon des critères psychomédicaux. Au contraire de l' infirme ou de l'inadapté qui renvoient à un vocabulaire « déceptif», le handicap réfère pour sa part à une compensation de l'inégalité des chances, par un dispositif légal, ou par des mesures d'accès à l'emploi ou encore d'accessibilité architecturale (Stiker, 2013).

Au-delà de cette terminologie, l'«infirmité» est alors appréhendée comme un problème à la fois social et médical, à la jonction entre la maladie chronique et un «état». Cette dualité s'exprime dans la diversité des acteurs qui interviennent auprès de cette clientèle - philanthropes, enseignants, travailleurs sociaux, médecins et chirurgiens - et dans le statut hybride des premiers services qui lui sont spécifiquement destinés sous la forme de l'«hôpital-école» (Byrom, 2001). Catégorie floue et mouvante, l'infirmité échappe à une définition claire et mesurable, une difficulté d'ailleurs soulevée par les philanthropes et les médecins ayant la tâche de circonscrire la population visée par leurs actions. Elle englobe diverses déficiences «orthopédiques» causées par des malformations congénitales, tel le pied-bot ou les scolioses, de même que des déformations liées à la tuberculose osseuse, ainsi que l'atrophie ou la paralysie des membres causée par le virus de la polio. Certaines pathologies comme le rachitisme ou la tuberculose des os sont de véritables «maladies de la misère», résultant de la malnutrition et de conditions de vie insalubres ${ }^{6}$. Ce lien entre les causes des déficiences et la pauvreté 
sera souligné par les philanthropes en vue d'insister sur le poids les facteurs socioéconomiques et de discréditer un autre discours désignant plutôt les tares héréditaires responsables de l'infirmité.

\section{Contexte d'une prise de conscience des droits des enfants handicapés, 1920-1940}

En Amérique du Nord, des associations philanthropiques destinées aux enfants infirmes moteurs se structurent dans les années 1920, au lendemain de la Première Guerre mondiale. Cette périodisation est sans doute liée à plusieurs facteurs, notamment les avancées médicales au lendemain de la Grande guerre qui permettent de sauver davantage de malades estropiés ou paralysés, conjugués à une sensibilité nouvelle face aux personnes handicapées, une nécessité de réparation après le charnier (Stiker, 2013). À la même époque, une première vague d'épidémie de poliomyélite, maladie très contagieuse demeurée à l'état endémique pendant des siècles, touche les États-Unis, atteignant alors un seuil de gravité sans précédent en 1916, alors qu'elle frappe frappant 27000 personnes en périphérie de New York, causant près de 6000 morts. Les enfants sont aussi visés au premier chef par les actions des réformateurs sociaux et des philanthropes qui sonnent l'alarme face aux conditions de vie déplorables des familles de la classe ouvrière et tentent de lutter contre les taux effarants de mortalité infantile. Une nouvelle mentalité à l'égard de l'enfance émerge au tournant du $20^{\mathrm{e}}$ siècle, contribuant à sa lente progressive «sacralisation» évoquée par Viviana Zelizer, (Zelizer, 1985). L'enfant est désormais perçu comme un être fragile à protéger, un futur citoyen plutôt qu'une main-d'œuvre bon marché. Cette nouvelle sensibilité va entraîner l'adoption de lois visant la protection des enfants et le développement de services qui leur sont spécifiquement destinés, notamment des soins pédiatriques.

Au niveau transnational, les états sonnent l'alarme face au trafic et à l'exploitation des enfants, ainsi qu'à la nécessité de les protéger en cas de conflits armés. Ainsi, en 1924, la Société des Nations (SDN) institue un Comité de la protection de l'enfance et adopte une Déclaration des droits de l'enfant. Selon Dominique Marshall, (Marshall, 2008), l'avènement de droits internationaux des enfants apparaît alors, aux yeux de ses défenseurs comme celle d'un «humanitarisme minimal», le gage des «promesses brisées de la politique internationale». La question particulière des droits des enfants infirmes ne 
figure pas dans cette déclaration, sinon de manière implicite à l'article 2 qui stipule que «l'enfant malade doit être soigné ; l'enfant arriéré doit être encouragé». De par les soins que sa condition exige et sa conceptualisation comme un être vulnérable, le jeune infirme se situe sans doute à la frontière entre ces deux catégories de l'enfance malade et «arriérée». En 1926, Charlotte Whitton, directrice du Conseil canadien pour la sauvegarde de l'enfance et représentante canadienne au Comité de protection de l'enfance (CPE) de la SDN, propose la tenue d'une enquête sur les problèmes des enfants estropiés sous la gouverne de cette instance internationale. En dépit de l'influence diplomatique de Whitton au sein du CPE (Marshall, 2000), cette demande est rejetée par le comité qui allègue la difficulté à circonscrire le sujet en raison de la définition floue du terme «estropié». Le CPE priorise alors la question des enfants aveugles qui forme un groupe plus restreint bénéficiant d'un solide appui médiatique lié au lobbying des associations dirigées par des aveugles (Brégain, 2018). En contrepartie, une enquête thématique plus limitée sur la formation et l'orientation professionnelle des enfants estropiés, à laquelle collabore Whitton, est tout de même confiée au Bureau international du travail. La SDN manifeste donc une certaine ouverture à la «cause» des enfants handicapés et elle sera interpelée par une association philanthropique afin d'adopter diverses mesures garantissant leurs droits.

\section{Un mouvement d'avant-garde : mobilisation internationale et adoption de la première charte des droits des enfants infirmes, 1922-1939}

En 1919, Edgar Allen, riche industriel américain, s'associe au club Rotary pour fonder une association d'assistance aux enfants infirmes, la Ohio Society for Crippled Children. Si cet organisme n'est pas le premier à offrir des services aux enfants handicapés moteurs, il se distingue par la volonté de son fondateur de porter la précarité des conditions de vie des enfants infirmes sur la place publique et de débattre de leurs droits au niveau national, puis sur la scène internationale. Personnage influent et charismatique, Allen convainc rapidement les rotariens des états voisins de mettre sur pied des associations pour enfants infirmes qu'il fédère en 1921 en une association nationale ${ }^{7}$. L'association milite pour un modèle d'intervention auprès des enfants infirmes financé par les gouvernements locaux, connu sous le nom de «Ohio Plan» (Floyd, 2011). Elle parvient ainsi à faire adopter des lois dans divers états américains 
contraignant les gouvernements à assurer des soins et une éducation aux enfants handicapés. En 1922, la Société ontarienne homonyme fondée la même année souhaite adhérer à la fédération qui devient dès lors l'International Society for Crippled Children (ISCC).

Le dynamisme du fondateur permet de tisser des liens avec plusieurs pays d'Europe et en 1929, une première conférence mondiale réunissant une cinquantaine de délégués provenant de douze pays se déroule à Genève. Au terme des discussions, l'assemblée adopte une série de résolutions, proclamant notamment que «tout estropié a le droit à l'égalité physique, mentale ou sociale ainsi qu'à une assistance en matière de soins et d'éducation de la part de son pays ou de son état» et que «l'assistance aux estropiés n'est pas seulement de responsabilité humanitaire, mais aussi de responsabilité sociale ${ }^{8}$. Ces résolutions annoncent avant 1 'heure un changement fondamental dans la manière de définir le devoir des sociétés face à leurs membres handicapés. Ainsi, au lieu de reléguer la prise en charge des jeunes infirmes aux initiatives charitables ou au domaine privé de la philanthropie, les déclarations de principes votées à Genève élargissent cette responsabilité à l'ensemble de la collectivité. Plutôt qu'être perçus comme des bénéficiaires plus ou moins passifs d'une action «humanitaire», les enfants infirmes sont au contraire conceptualisés comme de futurs citoyens jouissant à ce titre d'un ensemble de droits. Au-delà de ces affirmations générales, les participants de cette conférence demeurent cependant divisés en ce qui a trait à l'étendue et aux modes d'intervention de la part des pouvoirs publics. En France et en Allemagne, le modèle privilégié est davantage centralisé puisque l'État y finance de vastes institutions, alors qu'aux États-Unis la place prépondérante de la philanthropie privée est farouchement défendue. D'autres pays comme la Suède se démarquent par une combinaison de l'aide privée et publique selon une économie sociale mixte semblable au modèle canadien (Valverde, 1995). Autre sujet de discorde : le modèle de scolarisation à privilégier pour les enfants. Certains conférenciers optent pour les classes spéciales en milieu régulier, d'autres pour des internats et des écoles spéciales. Enfin, les délégués estiment que le manque flagrant de données relatives au recensement et au dépistage des cas d'infirmité constitue une lacune criante. À l'issue des travaux de cette conférence, une pétition soumise à la Société des Nations $(\mathrm{SDN})$ réclame la création d'un Bureau international 
d'information et de collecte de données. La nécessité de la prévention des infirmités et des malformations a été le seul sujet sur lequel l'unanimité des opinions a pu se réaliser, résume en conclusion le Dr Norman Carver du Comité central pour les estropiés à Londres ${ }^{9}$. L'ISCC publie d'ailleurs dès la fin des années 1920 des dépliants éducatifs visant à sensibiliser le grand public quant à la nécessité d'agir promptement pour éviter que certaines conditions ou affections non traitées ne dégénèrent en une incapacité permanente chez l'enfant. Ces membres de la bourgeoisie libérale visent les familles des classes populaires, critiquant leur prétendue négligence responsable de «fausses» infirmités et ne mâchant pas leur mot à l'égard de l'ignorance de certains parents ${ }^{10}$.

Une deuxième conférence de l'ISCC, réunissant essentiellement des philanthropes, des représentants du milieu médical (médecins, chirurgiens) et des directeurs d'écoles spécialisées, se déroule à La Haye en 1931. Si ce réseau est à forte prédominance masculine, certaines femmes en font partie, comme Bell Greve, une travailleuse sociale détenant aussi une formation d'avocate et directrice du Cleveland Rehabilitation Centre qui joue un rôle important dans le mouvement international de défense des droits, notamment à titre de secrétaire générale de l'ISCC dès $1939^{11}$. Parmi les sujets abordés par les conférenciers figure la nécessité de l'intégration sociale et les débouchés professionnels. Dans son discours inaugural, le président Edgar Allen réitère l'importance de coordonner le travail des divers organismes (hôpitaux écoles, dispensaires, maisons de convalescence) à travers un réseau de professionnels dûment formés dans les universités et soutenu par un financement adéquat. Rappelant l'importance de lutter contre les préjugés, il soutient : «the aim and purpose is that the cripple may not only regain control of his physical powers but acquire a new sense of his value». Si les jeunes handicapés doivent être convaincus de leur «valeur», ce n'est pas seulement en vue de regagner leur estime personnelle, mais aussi parce que pour les membres de l'élite libérale comme Allen, les enfants représentent un capital humain à secourir et encadrer en raison de leur potentiel futur pour l'avenir du pays. Au terme de la conférence, les délégués adoptent une «Charte des droits des enfants infirmes» qui se décline en dix articles. Le premier article porte sur l'importance de la prévention pré et post-natales ainsi qu'au moment de l'accouchement en vue de prévenir les malformations congénitales. Le second article proclame le droit des enfants de se développer «free from 
crippling conditions» : les facteurs socio-économiques comme la sous-alimentation et le manque d'hygiène sont pointés du doigt.

En dépit de ces précautions, il est impossible de prévenir toutes les infirmités, c'est pourquoi le $3^{\mathrm{e}}$ article préconise des soins le plus tôt possible aux tout-petits et de façon continue. Les articles subséquents promulguent le droit à l'éducation ainsi que le droit à une formation permettant aux futurs adultes de gagner leur vie et de prendre part à la vie active dans la société. L'article 8 affirme le droit pour les enfants d'être «traités avec considération», non seulement par les personnes en charge de leurs soins ou de leur bien-être, mais de la part de la société en général, et ce à l'abri des moqueries ou de la «pitié démoralisante» ${ }^{12}$. Ces résolutions sont adoptées à l'unanimité par l'assemblée au terme de la conférence. Le document est largement diffusé à travers le monde, incluant au Canada où des philanthropes sont sensibilisés à la cause.

\section{Participation québécoise au mouvement transnational de défense des droits, 1930-1939}

L'influence de ce mouvement de défense des droits des enfants infirmes s'étend au-delà de la frontière américaine jusqu'au Canada par le biais du milieu philanthropique anglophone. En 1930, des hommes d'affaires montréalais membres du club Rotary fondent la Société de secours aux enfants infirmes de la province de Québec (SSEIQ), calquée sur les associations homonymes de l'Ohio et de l'Ontario (Hanes, 2018). L'organisme vise à coordonner les divers services aux petits infirmes en agissant comme lien entre les institutions et les enfants, offrant une ébauche de service social à domicile et dépistant les enfants dans leur milieu. La direction est confiée à une «secrétaire exécutive» bilingue issue de la bourgeoisie libérale francophone, Marie-Alida Daigle. Afin de promouvoir le bien-être des enfants handicapés, l'organisme vise d'abord à leur fournir les soins dont ils ont besoin en les conduisant dans les divers hôpitaux de la ville. Outre le transport et un certain travail social, la SSEIQ procure gratuitement des appareils orthopédiques, elle organise aussi un camp d'été qui est perçu comme un prolongement des soins donnés dans les hôpitaux le reste de l'année. Ce modèle de services est fortement inspiré par la philanthropie anglophone qui instaure une assistance à domicile dans les «institutions sans murs» et privilégie le placement en familles d'accueil, tandis 
que les œuvres de charité franco-catholiques s'organisent davantage au sein des institutions dirigées par les communautés religieuses (Bourbeau, 2015). La SSEIQ se distingue aussi par son approche non confessionnelle puisqu'elle intervient aussi bien auprès de jeunes franco-catholiques que des anglophones protestants ou catholiques, de même que des enfants juifs, comme l'atteste la nomination de représentants de chacune des religions au sein de son conseil d'administration. Des cas provenant de régions éloignées de la province sont référés à la directrice M-A Daigle. Toutefois, on peut s'interroger sur le silence presque total à l'égard des enfants autochtones : mis à part quelques initiatives isolées, ces derniers ne semblent manifestement pas considérés comme de futurs citoyens à «guérir» pour assurer l'avenir de la nation ${ }^{13}$.

Dès sa fondation, la Société de secours aux enfants infirmes du Québec adhère à la fédération internationale dont elle reçoit les publications et accueille son président, Edgar Allen, lors d'une visite à Montréal en juin 1931 relatée dans les journaux anglophones de la métropole. Le quotidien The Gazette évoque aussi les démarches entreprises par Allen auprès de la SDN pour obtenir un bureau permanent d'information : un article paru le 17 mars 1930 souligne la difficulté de répertorier le nombre de cas dans chaque pays et la nécessité d'un travail concerté dépassant le cadre des frontières nationales ${ }^{14}$. Une des premières actions de la SSEIQ sera d'entreprendre dès 1931 un recensement des enfants infirmes physiques dans toute la province de Québec, ce qui allait justement dans le même sens que les priorités identifiées par la Société internationale lors de la Conférence de Genève de 1929. Si aucun de ses membres n'assiste officiellement aux premières conférences internationales de 1929 et 1931, la SSEIQ délègue son président en Ohio pour visiter les institutions spécialisées américaines et la directrice M-A Daigle à la conférence internationale de Londres en 1939. Outre ces philanthropes, le président de la Commission des écoles catholiques de Montréal, Victor Doré, s'intéresse de près aux initiatives internationales auprès des enfants infirmes puisqu'il cherche à justifier l'ouverture de classes spéciales pour les «anormaux» dans la métropole. Pour ce tenant de l'hygiène mentale, la séparation des élèves normaux et des arriérés ou des infirmes dans les écoles s'impose pour prévenir les désordres sociaux, mais surtout en vue d'accroître l'efficacité de l'école à former de futurs citoyens utiles. Ce comptable de profession partage avec les philanthropes de 
l'ISCC comme Edgar Allen une vision utilitaire de l'enfance. Selon Doré, la société ne peut se permettre de perdre ces forces vives, d'où l'importance de former les jeunes handicapés à des métiers adaptés à leur potentiel et qui leur permettront de contribuer activement à l'économie de la nation. Doré est envoyé en mission eu Europe afin de visiter les institutions les plus réputées pour jeunes infirmes, et il participe lui aussi aux congrès internationaux, en plus de devenir l'un des directeurs de l'ISCC. Un congrès annuel est même organisé à Montréal en 1934, sous la présidence conjointe de la SSEIQ et de Victor Doré, auquel assistent surtout des Américains, mais aussi quelques délégués français et britanniques ${ }^{15}$.

Au Québec, tout comme ailleurs en Occident, la période de l'entre-deux-guerres a donc été favorable à l'émergence d'un mouvement de défense des droits des enfants infirmes. Celui-ci constitue un vaste réseau formé de représentants de la plupart des pays d'Europe de l'Ouest ainsi que des États-Unis et du Canada, et même de certains pays d'Amérique latine. Réunissant parmi ses membres des philanthropes, des éducateurs et des médecins, il insiste sur la prévention des causes d'infirmité et cible les facteurs socioéconomiques responsables de l'infirmité des enfants, véhiculant donc une représentation éminemment sociale du handicap. Contrairement au modèle médical qui s'impose après la Deuxième Guerre mondiale, ce mouvement défend l'idée que la réhabilitation des jeunes handicapés repose, non pas seulement sur leurs épaules, mais sur des changements externes, notamment l'accès à une formation et la disparition des préjugés entravant leur participation au marché du travail. Néanmoins, en dépit de son accent sur les mesures sociales et législatives en faveur des jeunes handicapés, ce mouvement est aussi empreint de contradictions qui s'expriment dans l'objectif ultime visé, celui de parvenir à éradiquer l'infirmité (the eradication of crippledom), comme l'illustre la figure 1. 


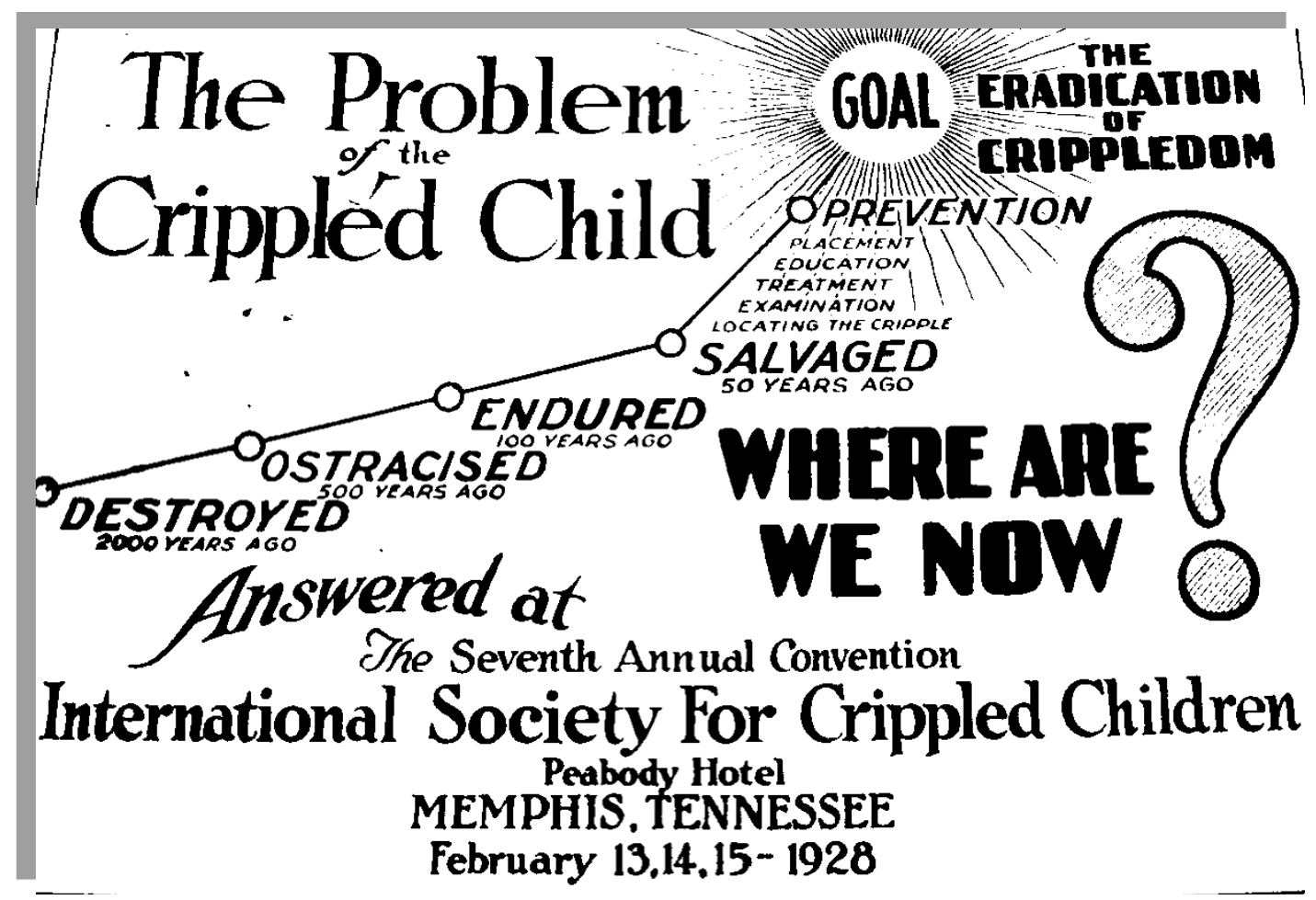

Figure 1: Légende: «The Problem of the Crippled Child», 7e assemblée annuelle de l'ISCC, 1928. À l'extrémité de la ligne du temps se trouve énoncé l'objectif ultime: The eradication of Crippledom.

Cette courbe temporelle schématise la progression des attitudes sociétales à l'égard des enfants infirmes depuis l'Antiquité, où ils étaient souvent condamnés à périr, jusqu'au Moyen-Âge où ils étaient systémtiquement ségrégués, pour ensuite être tolérés et sauvés à l'ère industrielle. Le plan d'action de l'ISCC est résumé dans la dernière portion de la figure : dépister, diagnostiquer, traiter, éduquer et placer les jeunes en emploi. La prévention de tous les cas doit permettre, en bout de parcours, la «suppression radicale et définitive de la classe des estropiés», tel que le décrit la traduction française de la conférence d'Edgar Allen à La Haye ${ }^{16}$. Ce discours est empreint d'une idéologie de la guérison, reflétant une confiance triomphaliste et utopique à l'égard du progrès inébranlable de la science devant permettre à terme la disparition de l'infirmité. Mais elle ouvre aussi la porte à des interprétations chargées d'intolérance et de capacitisme (Parent, 2017), en faisant valoir que l'idéal pour une société est l'absence de la «classe des estropiés». Alors que les délégués de la conférence proclament que «tout estropié a le droit d'attendre de son pays l'égalité physique, mentale et sociale», ils tiennent paradoxalement un double discours teinté d'eugénisme en faisant la promotion des 
mesures prophylactiques, décrites comme le «remède» permettant d'éradiquer l'infirmité. Le dépistage précoce et la déclaration obligatoire des cas à la naissance sont notamment présentés comme une panacée en vue de diminuer le nombre d'infirmes ${ }^{17}$.

$\mathrm{Au}$ final, la formation d'un Bureau tout comme l'adoption de la charte des droits des enfants infirmes à la Société des Nations demeurent lettre morte, notamment en raison de la conjoncture politique internationale : la montée du fascisme dans les années 1930 entraîne le retrait de plusieurs pays de la SDN qui s'avère incapable de prévenir le déclenchement de la Deuxième Guerre mondiale. Selon Gildas Brégain, (Brégain, 2018), cet échec est aussi lié à la faible avancée des législations nationales en la matière et à l'incapacité de l'ISCC à susciter des débats internationaux de qualité sur les aspects législatifs. La Société internationale des enfants infirmes cesse pratiquement toute activité après le congrès de Londres de 1939 et ce pendant plusieurs années.

\section{Un second souffle au réseau transnational de défense des droits : de l'enfance infirme à l'enfance "exceptionnelle», 1950-1970}

Après la Deuxième Guerre mondiale, avec la création de l'Organisation des Nations unies, le réseau international d'échanges entre les promoteurs des droits des personnes handicapées connaît un second souffle, sous la gouverne de Bell Greve qui devient la secrétaire générale de l'ISCC. En 1950, une réunion internationale est organisée à Genève sous les auspices de l'UNESCO sur les «problèmes éducatifs des enfants infirmes moteurs». Les experts réunis sous la présidence de Jean Piaget adoptent certaines résolutions, proclamant notamment la nécessité urgente d'une meilleure coordination entre les programmes éducatifs et les traitements, en vue de «permettre à l'enfant infirme moteur de bénéficier des meilleures possibilités d'éducation conformes à ses talents ${ }^{18}$. Marie-Alida Daigle assiste à cette important congrès comme déléguée du Canada. À son retour elle fait part de ses impressions lors d'une conférence de presse, soulignant, non sans un certain chauvinisme, l'avance des institutions montréalaises sur plusieurs organismes européens ${ }^{19}$.

L'implication de l'UNESCO dans la sphère de l'enfance handicapée demeure toutefois limitée, comme l'étudie Gildas Brégain (Brégain, 2018), qui en analyse les conséquences : d'une part, ce faible engagement de l'UNESCO contribue à la domination exclusive du secteur de la réadaptation sur le secteur de l'éducation spécialisée qui 
devient une des composantes des programmes de réadaptation, et d'autre part, le retrait de cette organisation non confessionnelle entraîne la prédominance de normes fortement influencées par l'Église catholique. Ainsi, dans les décennies 1950 et 1960, les spécialistes québécois de l'enfance inadaptée partagent plutôt leurs connaissances lors des congrès organisés par le Bureau international catholique de l'enfance (BICE), (Ferretti, Bienvenue, 2010). En 1957, le congrès du BICE se déroule d'ailleurs au Québec, dans les villes de Montréal et Trois-Rivières. Lors de son VIIIe congrès à Beyrouth en 1963, le BICE adopte une série de «vœux et principes» qui constituent une véritable proclamation des droits des enfants «inadaptés». Plusieurs de ces principes font référence à l'importance de l'intégration des enfants et de leur développement hors d'un cadre institutionnel, ce qui reflète les préoccupations grandissantes pour la psychopédagogie au début des années 1960. La dimension affective, le caractère unique de chaque enfant, l'individualité du développement et du cheminement de l'enfant vers l'autonomie caractérisent dorénavant les principes éducatifs (Brabant, 2006). Ces valeurs ont une résonance pour les enfants en difficulté puisqu'elles confirment le «droit de l'enfant à sa famille», et désavouent leur institutionnalisation, comme en témoignent les principes ci-dessous :

Aucun groupe d'enfants inadaptés ne doit faire l'objet d'une politique systématique d'institutionnalisation. L'enfant inadapté a droit à sa famille. L'aide que l'État se doit d'apporter à celle-ci n'aboutira pas à lui faire perdre le sens de ses responsabilités et à la déposséder de son autorité naturelle. $\mathrm{Au}$ contraire, on développera les solutions d'assistance éducative et de rééducation à domicile ou en externat. Dans les internats, on recherchera des formules à petits effectifs et du type familial (Principe V) ${ }^{20}$.

Tout comme la première «vague» de lutte pour la défense des droits, cette seconde mobilisation est largement portée par les éducateurs et divers spécialistes au nom des enfants, cette situation étant appelée à changer. En effet, dans les années 1970, les parents québécois prennent le devant de la scène et s'organisent en associations militantes, de concert avec les activistes en situation de handicap, pour revendiquer des services et l'adoption de lois assurant l'exercice de droits. Cet activisme par les personnes 
concernées en leur nom propre contribue à éclipser les associations philanthropiques comme la Société de secours (SSEIQ). En outre, les gouvernements fédéraux puis provinciaux agissent en adoptant des politiques sociales à l'égard des personnes démunies. De nouveaux acteurs sociopolitiques jouent donc un rôle accru, avec la montée de l'État providence québécois. Au début des années 1960, le gouvernement provincial organise de vastes commissions d'enquête sur l'éducation et la santé et intervient massivement dans tous les secteurs de la société québécoise en vue d'assurer son «virage» vers la modernité. Dans les années 1970, les principes de la normalisation et de l'intégration scolaire influencent fortement les politiques adoptées par les spécialistes de l'enfance inadaptée. Au cours de cette décennie, les intervenants tentent de démontrer la primauté de l'enseignement sur le secteur «médico-psychiatrique» et de renverser la manière de diagnostiquer les enfants, fondée sur le «modèle médical» et jugée discriminante. Ajouter gilles

\section{De l'enfant à protéger à l'enfant sujet de droits : troisième vague de mobilisation et émergence du handicap comme enjeu sociopolitique, 1960-1980}

Le passage de la protection à l'exercice des droits des personnes invalides, y compris les enfants « inadaptés », est intimement lié à l'apparition du concept du handicap, dont l'utilisation se répand à partir des années 1970. Dans la perspective de ceux qui souhaitent imposer cette nouvelle donne, les enfants handicapés au Québec cessent d'être des êtres vulnérables à protéger et deviennent des sujets de droits, un changement de perception reflété par le discours médiatique et entériné par certaines mesures législatives. Ainsi, en 1975, dans un éditorial virulent, la journaliste Françoise Gélinas scande : « Nos enfants handicapés ne devraient pas mendier ce qui leur revient de droit $»^{21}$. Dans ce texte, elle s'insurge de la façon dont la Société de secours s'y prend pour mousser sa campagne de souscription, en couronnant une « petite reine » des enfants infirmes et en «l'exposant » à de multiples séances photographiques avec des personnalités politiques et des journalistes. Les propos de l'éditorialiste mettent en évidence le clivage entre la promotion des droits des enfants que prétend défendre l'association philanthropique et l'image sensationnaliste véhiculée à travers ses campagnes de souscription. Plutôt qu'être l'objet de sollicitude et de la générosité des 
adultes, les jeunes handicapés devraient avoir accès à des services selon le principe de l'égalité des chances pour tous, insiste Gélinas qui reprend le message véhiculé par le mouvement de défense des droits par et pour les personnes handicapées elles-mêmes qui s'intensifie au tournant des années 1980.

Les acteurs de ce mouvement de mobilisation sans précédent entendent bien être pris en compte par les décideurs politiques. Ainsi, au Québec, peu après le dépôt du projet de loi sur la « protection des personnes handicapées », le Comité de liaison des handicapés physiques du Québec est créé pour recueillir les recommandations de divers groupes et un front commun est formé qui publie un manifeste le 25 octobre 1977. Des associations de parents d'enfants handicapés, comme l'Association de la paralysie cérébrale du Québec, s'associent à ce mouvement. Le milieu associatif se transforme et ses revendications contribuent à faire du handicap un phénomène politique (Boucher, 2005). Dans cette mouvance, l'Assemblée nationale du Québec adopte, en 1978, la Loi assurant l'exercice des droits des personnes handicapées. Cette dernière modifie la charte québécoise des droits et libertés de la personne de 1975 en ajoutant le handicap et tout moyen de pallier un handicap comme motif éventuel de discrimination. La loi a également pour effet de créer l'Office des personnes handicapées du Québec (OPHQ). Cette mobilisation trouve un écho sur la scène internationale : l'ONU décrète 1981 «Année internationale des personnes handicapées », ce qui permet de renforcer le mouvement de prise de parole amorcé précédemment.

En parallèle, à la fin des années 1970, le mouvement de défense des droits des personnes handicapées, qui s'appuie sur le principe de la normalisation, intensifie ce mouvement de maintien des jeunes déficients dans leur milieu familial. Dans la mouvance de la désinstitutionnalisation, l'État québécois instaure une série de mesures qui favorisent cette prise en charge des familles, notamment par la bonification des allocations familiales dans le cas de jeunes ayant une incapacité. En 1985, dans la foulée des revendications de l'Année internationale des personnes handicapées, le gouvernement du Québec adopte la politique $A$ part... égale. Misant sur la prévention des déficiences et l'intégration sociale des personnes handicapées, cette politique ambitieuse présente des mesures de soutien aux familles propices à l'intégration d'un enfant handicapé. Elle demande notamment la reconnaissance du rôle joué par un des parents (la mère le plus 
souvent) qui assume des responsabilités équivalentes à un emploi (OPHQ, 1984). Toutefois, dans un contexte de crise économique qui sévit au début des années 1980, l'État renégocie sa part de responsabilités à l'égard des personnes vulnérables et tend à en faire porter la charge sur les familles. Le secteur communautaire offre alors des services de plus en plus essentiels afin de soulager les familles.

Ainsi, confrontées aux manques de ressources leur offrant des services de répit temporaire, des familles se regroupent en 1983 et fondent l'association Solidarité de parents de personnes handicapées physique-moteur (SPPH). L'association se caractérise par une mobilisation de militants endossant une vision contestataire puisqu'elle adhère au «modèle social du handicap » et tend à faire de celui-ci un enjeu sociopolitique. Outre les services de répit, de soutien et d'accompagnement aux familles, l'organisme milite en vue de favoriser l'intégration des jeunes bambins dans les garderies régulières, un dossier qui mobilise les parents au milieu de la décennie ${ }^{22}$. Les parents exercent des pressions auprès des décideurs politiques afin de les inciter à mettre sur pied divers services ou encore à mieux financer les initiatives des associations communautaires, comme le gardiennage ou le répit-dépannage. Dans un contexte de désengagement progressif de l'État au cours des années 1980, ces services offerts par le secteur communautaire deviennent essentiels pour le maintien de l'enfant handicapé dans son milieu, une situation qui touche $99 \%$ des familles québécoises au lendemain des vagues de désinstitutionnalisation (Tétrault, Blanchette, 1991). Investies du rôle de proche aidante (caring role) et de première « intervenante » dans la vie de leur enfant, les mères en particulier prolongent ces responsabilités dans la sphère publique, interpelant ainsi les gouvernements en vue de modifier les politiques sociales à l'égard des jeunes handicapés.

\section{Conclusion}

Nombre d'ouvrages d'intellectuels militants en situation de handicap réfèrent à deux périodes dans l'histoire du handicap : un «avant» les années 1960-1970 où le modèle médical a largement dominé et un «après», alors que l'émergence de la lutte des droits et la prise de parole par les personnes handicapées elles-mêmes ont permis une transition du modèle médical au modèle social ${ }^{23}$. Notre étude nous amène à questionner cette perspective. Cette recherche a en effet tenté de montrer que le passé des enfants handicapés au Québec ne suit pas une progression linéaire menant de la stigmatisation au 
plein exercice des droits, mais il est plutôt traversé d'avancées, suivies de régressions. En effet, les premières décennies du $\mathrm{XX}^{\mathrm{e}}$ siècle sont plutôt caractérisées par une prédominance de la dimension socio-pédagogique et par un éveil des consciences qui entraîne la naissance d'un mouvement de défense des droits, aux accents fortement paternalistes et condescendants il est vrai (y compris chez les femmes philanthropes d'ailleurs), mais s'opposant dans l'ensemble à d'autres perceptions courantes au cours de l'entre-deux-guerres, telles les théories eugénistes. Les préoccupations des philanthropes à l'égard des conditions de vie précaires des enfants handicapés ont été portées sur la scène internationale par un lobbying à la Société des Nations. La charte des droits des enfants infirmes, même si elle est demeurée lettre morte, constitue un projet d'avantgarde puisque les principes mêmes du mouvement d'intégration sociale des personnes handicapées s'y trouvent exprimés, quarante ans avant l'heure. Dans les années 1950 et 1960, la mobilisation internationale connaît un second souffle à travers les réseaux des spécialistes de l'enfance «inadaptée», avant d'être portée par la voix des parents et des personnes handicapées elles-mêmes au tournant des années 1980, ce qui crée des changements législatifs et ouvre l'année internationale des personnes handicapées en 1981.

En dépit d'avancées majeures lors des décennies 1980 et 1990, le désengagement de l'État néo-libéral, le néo-eugénisme sous la forme du dépistage systématique des anomalies intra-utérines, la pression de la performance et l'intolérance à la différence dans les écoles freinent l'entière participation des jeunes en situation de handicap à la société québécoise. En outre, le discours capacitiste qui met l'accent sur la vulnérabilité, le sentimentalisme ou l'idéologie de la guérison continue à perpétuer une fausse image des enfants handicapés, refusant de les reconnaître pleinement et tout simplement comme de véritables sujets de droits.

\section{Remerciements:}

Cette recherche a bénéficié du soutien financier du Fonds de recherche du Québec - Société et culture (FRQSC) que je remercie, ainsi que Dominique Marshall pour sa lecture d'une version préliminaire de l'article. 
${ }^{1}$ Pour des études historiques intégrant cette approche, voir notamment Paul Longmore et Lauri Umansky, dir., The New Disability History: American Perspectives, New York, New York University Press, 2001; Susan Burch and Ian Sutherland, "Who's Not Yet Here? American Disability History », Radical Historical Review, vol. 2006, no 94 (2006) p. 127-147; Jay Dolmage, «Disabled Upon Arrival: The Rhetorical Construction of Disability and Race at Ellis Island », Cultural Critique, vol. 77, no 1 (2011), p. 24-69.

${ }^{2}$ Gildas Brégain, Pour une histoire du handicap au XXe siècle. Approches transnationales (Europe et Amériques, Rennes, PUR, 2018, p. 22.

${ }^{3}$ Cette association se nommait au moment de sa fondation en 1930 la Société de secours aux enfants infirmes de la province de Québec (SSEIQ). Elle devient la Société pour les enfants handicapés dans les années 1970, pour être rebaptisée «Fondation Papillon» en 2017.

${ }^{4}$ Le modèle social du handicap s'affirme à partir des années 1970 en opposition à l'approche biomédicale qui fait porter le poids de l'incapacité sur l'individu. Proposant un changement de paradigme radical, le modèle social, lié à la mobilisation des activistes handicapés, dénaturalise le handicap en le conceptualisant comme une construction sociale et culturelle. Voir notamment Normand Boucher, «Handicap, recherche et changement social. L'émergence du paradigme émancipatoire dans l'étude de l'exclusion sociale des personnes handicapées», Lien social et Politiques, no 50 (2003), p. 147-164. Sur la littérature anglosaxonne, Tom Shakespeare et al., "The social model of disability», The Disability Studies Reader, vol. 2 (2006), p. 197-204.

${ }^{5}$ Le préfixe in accolé au mot latin firmus (ferme, fort) traduit la négation de la force. Les infirmes sont donc des êtres faibles à secourir par la charité, comme l'étudie Henri-Jacques Stiker, "Comment nommer les déficiences ?», Ethnologie française, 2009/3, tome XXXIX, p. 464.

${ }^{6} \mathrm{La}$ tuberculose osseuse se transmettait chez les enfants par le lait non pasteurisé ou par la viande infectée par la souche bovine de la maladie. Le rachitisme est dû à une carence en calcium, en magnésium et en vitamine D entraînant des déformations du squelette. Ces maladies font de véritables ravages chez les enfants indigents jusqu'aux années 1940. Voir Katherine McCuaig, The Weariness, the Fever and the Fret. The Campaign against Tuberculosis in Canada, 1900-1950, Montréal et Kingston, McGill-Queen's University Press, 1999; Martin Tétreault, «Les maladies de la misère — aspects de la santé publique à Montréa 1-1880-1914», Revue d'histoire de l'Amérique française, vol. 36, no 4 (1983), p. 507-526.

${ }^{7}$ Nora Groce, From Charity to Disability Rights. Global Initiatives of Rehabilitation International, 19222002, New York, Rehabilitation International, 2002, p. 5-6.

${ }^{8}$ ACSDM, Fonds service des études, bureau de l'enseignement aux élèves physiquement handicapés, (ciaprès SÉ-ÉH), «Rapport de Victor Doré, délégué à la Conférence mondiale de La Haye », 1931 (nous soulignons).

${ }^{9}$ ACSDM, SÉ-ÉH, 16A, «Rapport de Victor Doré délégué à la Conférence mondiale de La Haye», 1931.

${ }^{10}$ «Crippling is largely preventable. Let's prevent it», dépliant ISCC, 1929.

11 Nora Groce, «Rehabilitation in an Historic Perspective: The Work of Bell Greve», Journal of Rehabilitation, 26, 1, 1993, p. 10.

${ }^{12}$ ASSEIQ, The Crippled Child's Bill of Rights, International Society for Crippled Children, 1931.

${ }^{13}$ En 1952, de jeunes Cris ayant contracté la polio sont transportés de la Baie James à Montréal avec le concours de la SSEIQ et hospitalisés au Children's Hospital pendant plusieurs mois. Ces déplacements d'enfants issus des Premières nations au «Sud», souvent sans l'accord de leurs parents, sont révélateurs de l'attitude colonialiste et du triomphalisme scientifique qui prévalent sur le respect des cultures traditionnelles de ces «protégés». ASSEIQ, «Journey’s End», The Montreal Star, 31 janvier 1952.

${ }^{14}$ ASSEIQ, «Problem of Crippled Children's Welfare is International in Aspect», Montreal Gazette, 17 mars 1930; «Work on Crippled Children Takes on International Air: Situation Now Being Studied by League of Nations», Montreal Gazette, 10 juin 1931.

${ }^{15}$ ASSEIQ, L'œuvre internationale des enfants infirmes, Treizième congrès annuel, Montréal, 20 au 23 mai 1934.

${ }^{16}$ ACSDM, SÉ-ÉH, 16A, «Developing the World Movement», conférence d'Edgar Allen à La Haye. Annexe au rapport de Victor Doré», 1931.

${ }^{17}$ ACSDM, SÉ-ÉH, 16A, «Developing the World Movement...», 1931.

18 ASSEIQ, «Conférence d'experts sur les problèmes éducatifs des enfants infirmes moteurs : projet de résolution», Genève, 20-25 février 1950.

${ }^{19}$ Madeleine Towner, «Nos enfants handicapés bien traités», La Presse, 3 avril 1950. 
${ }^{20}$ «Congrès mondial sur les droits de l'enfant-Commission sur les droits des enfants inadaptés, Beyrouth avril 1963», cité dans Orientation pour une politique de l'enfance inadaptée, Gouvernement du Québec, 1969.

${ }^{21}$ ASSEIQ, Françoise Gélinas, « Nos enfants handicapés ne devraient pas mendier ce qui leur revient de droit », Le Journal de Montréal, 21 septembre 1975.

${ }^{22}$ Aujourd'hui Solidarité de parents de personnes handicapées. Archives SPPH.

\section{Références bibliographiques :}

Boucher, Normand. (2005). « La régulation sociopolitique du handicap au Québec », Santé, Société et solidarité, 2, 145-154.

Bourbeau, Amélie. (2015). Techniciens de l'organisation sociale. La réorganisation de l'assistance catholique privée à Montréal (1930-1974), Montréal et Kingston: McGillQueen's University Press.

Bourgault, Gilles. (2018). Entre revendication et partenariat: la construction du mouvement de défense des droits des personnes handicapées au Québec entre 1975 et 1985. Bulletin d'histoire politique, 27(1), 112-134.

Brabant, Louise. (2006). "L'enfance sous le regard de l'expertise médicale : 1930$1970 »$, Recherches sociographiques, 47, 2, 277-298.

Brégain, Gildas. (2018). Pour une histoire du handicap au XXe siècle, approches transnationales (Europe et Amériques). Rennes : PUR.

Byrom, Brad. (2001). «A Pupil and a Patient. Hospital-Schools in Progressive America », dans Paul Longmore et Lauri Umansky, dir., The New Disability History, New York: New York University Press.

Cooter, Roger. (2003). "The Disabled Body», dans R.Cooter et J. Pickstone, éds, Companion To Medicine in the Twentieth Century, Londres et New Yord: Routledge.

Droux, Joëlle. (2011). L'internationalisation de la protection de l'enfance: acteurs, concurrences et projets transnationaux (1900-1925). Critique internationale, (3), 17-33.

Ferretti, Lucia et Louise Bienvenue (2010). «Le Bureau international catholique de l'enfance : réseau et tribune pour les spécialistes québécois de l'enfance en difficulté (1947-1977) », Revue d'histoire de l'enfance "irrégulière » : Le Temps de l'histoire, 12, $155-176$.

Floyd, Barbara. (2011). «The Boy Who Changed the World: Ohio and the Crippled Children's Movement», Ohio History, 72-90.

Gardou, Charles. (2005). «Y a-t-il de l'universel dans ce tellement singulier?», Reliance, 1, 10-15. 
Groce, Nora. (2014). « Disability and the League of Nations : the Crippled Child's Bill of Rights and a Call for an International Bureau of Information, 1931 », Disability and Society, 29,(4), 503-515.

Groce, Nora. (2002). From Charity to Disability Rights. Global Initiatives of Rehabilitation International, 1922-2002, New York: Rehabilitation International.

Hanes, Roy. (2018). "Service Clubs and the Emergence of Societies for Crippled Children in Canada: The Rise of the Ontario Society for Crippled Children, 1920-1940», dans N. Hansen et al. dir., Untold Stories: A Canadian Disability History Reader, Toronto et Vancouver: Canadian Scholars.

Marshall, Dominique. (2008). «Dimensions transnationales et locales de l'histoire des droits des enfants. La Société des Nations et les cultures politiques canadiennes, 19101960 », Genèses, 2, 47-63.

Marshall, Dominique. (2000). «Tensions nationales, ethniques et religieuses autour des droits des enfants: la participation canadienne au Comité de protection de l'enfance de la Société des Nations», Lien social et Politiques -RIAC, 44, 101-123.

Office des personnes handicapées du Québec. (1984). A part...égale. L'intégration sociale des personnes handicapées : un défi pour tous, Drummondville : OPHQ.

Parent, Laurence. (2017). «Ableism/disablism, on dit ça comment en français?». Canadian Journal of Disability Studies, vol. 6, (2), 183-212.

Saunier, P. Y. (2008). Les régimes circulatoires du domaine social 1800-1940: projets et ingénierie de la convergence et de la différence. Genèses, (2), 4-25.

Stiker, Henri-Jacques. (2013). Corps infirmes et sociétés: Essais d'anthropologie historique.,Paris: Dunod.

Tétrault, Sylvie et Linda Blanchette. (1991). «Réflexion sur la condition de la mère de l'enfant handicapé : une intervention féministe à développer », Service social, 40, (2), 117-143.

Valverde, Mariana. (1995). «La charité et l'État : un mariage mixte centenaire », Lien social et politiques -RIAC, 33, 27-35.

Zelizer, Viviana. (1985). Pricing the Priceless Child: The Changing Social Value of Children, New York: Basic Books. 